\title{
ON THE COEFFICIENTS OF THE MINIMAL POLYNOMIALS OF GAUSSIAN PERIODS
}

\author{
S. GUPTA AND D. ZAGIER
}

\begin{abstract}
Let $l$ be a prime number and $m$ a divisor of $l-1$. Then the Gauss period $\omega=\zeta+\zeta^{\lambda}+\zeta^{\lambda^{2}}+\cdots+\zeta^{\lambda^{m-1}}$, where $\zeta=e^{2 \pi i / l}$ and $\lambda$ is a primitive $m$ th root of unity modulo $l$, generates a subfield $K$ of $\mathbb{Q}(\zeta)$ of degree $(l-1) / m$. In this paper we study the reciprocal minimal polynomial $F_{l, m}(X)=N_{K / \mathbb{Q}}(1-\omega X)$ of $\omega$ over $\mathbb{Q}$. It will be shown that for fixed $m$ and every $N$ we have $F_{l, m}(X) \equiv\left(B_{m}(X)^{l} /(1-m X)\right)^{1 / m}\left(\bmod X^{N}\right)$ for all but finitely many "exceptional primes" $l$ (depending on $m$ and $N$ ), where $B_{m}(X) \in \mathbb{Z}[[X]]$ is a power series depending only on $m$. A method of computation of this set of exceptional primes is presented. The generalization of the results to the case of composite $l$ is also discussed.
\end{abstract}

\section{Statement of Results}

Let $l$ be an odd prime number ${ }^{1}$ and $l-1=m \cdot d$ a decomposition of $l-1$ into positive factors. Then there is a unique cyclic extension $K_{d} / \mathbb{Q}$ of degree $d$ ramified only at $l$. It is contained in the cyclotomic field $\mathbb{Q}(\zeta)(\zeta=$ primitive lth root of unity) and is generated over $\mathbb{Q}$ by the Gaussian period

$$
\omega=\operatorname{Tr}_{\mathbb{Q}(\zeta) / K_{d}}=\zeta+\zeta^{\lambda}+\zeta^{\lambda^{2}}+\cdots+\zeta^{\lambda^{m-1}},
$$

where $\lambda \in(\mathbb{Z} / l \mathbb{Z})^{\times}$is a primitive $m$ th root of unity modulo $l$. The minimal polynomial of $\omega$,

$$
f_{l, m}(X)=\prod_{r \in \mathscr{R}}\left(X-\left(\zeta^{r}+\zeta^{\lambda r}+\zeta^{\lambda^{2} r}+\cdots+\zeta^{\lambda^{m-1} r}\right)\right),
$$

where $\mathscr{R}$ denotes a set of coset representatives for $(\mathbb{Z} / l \mathbb{Z})^{\times}$modulo $\langle\lambda\rangle$, gives an explicit irreducible polynomial of degree $d$ with cyclic Galois group and discriminant a power of $l$. We include $l$ and $m$ rather than $l$ and $d$ into the notation because we will be studying the coefficients of these polynomials for $m$ fixed and $l$ varying. Specifically, we will show that for $m$ and $n$ fixed the $n$th coefficient "from the end" of $f_{l, m}(X)$ is a polynomial in $l$ for all but finitely many "exceptional" primes $l$, and we will describe the computation of this polynomial and of the set of exceptional primes. The statement about the $n$th coefficient being a polynomial in $l$ for $l$ large, and some of our other

Received by the editor August 19, 1991 and, in revised form, January 3, 1992.

1991 Mathematics Subject Classification. Primary 11L05, 11T22; Secondary 11Y40.

${ }^{1}$ The case of composite $l$ will be considered briefly in $\S 5$. 
results, were first proved by Gurak [1, 2]; we will give a detailed comparison with Gurak's work at the end of $\S 1$.

To state the basic result conveniently, we turn $f_{l, m}(X)$ around, setting

$$
F_{l, m}(X)=X^{d} f_{l, m}\left(X^{-1}\right)=\prod_{r \in \mathscr{R}}\left(1-\left(\zeta^{r}+\zeta^{\lambda r}+\zeta^{\lambda^{2} r}+\cdots+\zeta^{\lambda^{m-1} r}\right) X\right) .
$$

Clearly, this reciprocal polynomial also has cyclic Galois group and generates the field $K_{d}$.

Theorem 1. For each integer $m \geq 1$ there exist power series $A_{m}(X), B_{m}(X) \in$ $\mathbb{Z}[[X]]$ related to one another by

$$
B_{m}(X)=(1-m X) A_{m}(X)^{m}
$$

such that for each natural number $N$ the congruence

$$
F_{l, m}(X) \equiv A_{m}(X) B_{m}(X)^{(l-1) / m} \quad\left(\bmod X^{N}\right)
$$

holds for all but finitely many primes $l \equiv 1(\bmod m)$.

Here, "all but finitely many" means all primes not belonging to a computable finite set depending only on $m$ and $N$. The computation of $A_{m}$ and $B_{m}$ will be given in $\S 2$. The beginnings of these power series for $m \leq 10$ are given in Table 1. Theorem 1 implies that for fixed $m$ and $n$ the $n$th coefficient of $F_{l, m}(X)$ is a polynomial of degree $\leq n$ in $l$ except for a finite number of exceptional primes $l$. If $m$ is a power of a prime number $p$, then $B_{m}(X)$ is in fact a power series in $X^{p}$, as we can see in Table 1 and will prove in Theorem 2 . In this case it follows that the power series in question has degree at most

\begin{tabular}{|c|c|c|c|c|c|c|c|c|c|c|c|}
\hline & 1 & $X$ & $X^{2}$ & $X^{3}$ & $X^{4}$ & $X^{5}$ & $X^{6}$ & $X^{7}$ & $X^{8}$ & $X^{9}$ & $X^{10}$ \\
\hline$A_{1}(X)$ & 1 & 1 & 1 & 1 & 1 & 1 & 1 & 1 & 1 & 1 & 1 \\
\hline$B_{1}(X)$ & 1 & 0 & 0 & 0 & 0 & 0 & 0 & 0 & 0 & 0 & 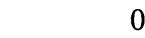 \\
\hline$A_{2}(X)$ & 1 & 1 & 1 & 2 & 3 & 6 & 10 & 20 & 35 & 70 & 126 \\
\hline$B_{2}(X)$ & 1 & 0 & -1 & 0 & -1 & 0 & -2 & 0 & -5 & 0 & -14 \\
\hline$A_{3}(X)$ & 1 & 1 & 2 & 4 & 11 & 29 & 73 & 207 & 574 & 1542 & 4435 \\
\hline$B_{3}(X)$ & 1 & 0 & 0 & -2 & 0 & 0 & -13 & 0 & 0 & -158 & 0 \\
\hline$A_{4}(X)$ & 1 & 1 & 2 & 7 & 21 & 77 & 257 & 963 & 3377 & 12816 & 46240 \\
\hline$B_{4}(X)$ & 1 & 0 & -2 & 0 & -7 & 0 & -50 & 0 & -456 & 0 & -4728 \\
\hline$A_{5}(X)$ & 1 & 1 & $\overline{3}$ & 11 & $\overline{44}$ & 180 & 796 & 3532 & 15906 & 72490 & 331282 \\
\hline$B_{5}(X)$ & 1 & 0 & c & 0 & 0 & -24 & 0 & 0 & 0 & 0 & -11052 \\
\hline$A_{6}(X)$ & 1 & 1 & 3 & 14 & 66 & 335 & 1736 & 9227 & 49744 & 271647 & 1497407 \\
\hline$B_{6}(X)$ & 1 & 0 & -3 & -4 & -18 & -60 & -269 & -1152 & -5412 & -25580 & -125478 \\
\hline$A_{7}(X)$ & 1 & 1 & 4 & 20 & 110 & 638 & 3828 & 23412 & 146865 & 930385 & 5955040 \\
\hline$B_{7}(X)$ & 1 & 0 & 0 & 0 & 0 & 0 & 0 & -720 & 0 & 0 & 0 \\
\hline$A_{8}(X)$ & 1 & 1 & $\overline{4}$ & 25 & 152 & 1034 & 6981 & 49554 & 350709 & 2553004 & 18557553 \\
\hline$B_{8}(X)$ & 1 & 0 & -4 & 0 & -34 & 0 & -696 & 0 & -19795 & 0 & -672916 \\
\hline$A_{9}(X)$ & 1 & 1 & 5 & 31 & 221 & 1637 & 12510 & 98618 & 789167 & 6394033 & 52492327 \\
\hline$B_{9}(X)$ & 1 & 0 & c & -6 & 0 & 0 & -387 & 0 & 0 & -68726 & 0 \\
\hline$A_{10}(X)$ & 1 & 1 & 5 & 38 & 289 & 2416 & 20428 & 179188 & 1587720 & 14328461 & 130327089 \\
\hline$B_{10}(X)$ & 1 & 0 & -5 & 0 & -55 & -48 & -1500 & -3360 & -58450 & -214560 & -2809859 \\
\hline
\end{tabular}
$[n / p]$ rather than $n$ and in particular is actually a constant for $n<p$, is linear

TABle 1. Coefficients of $A_{m}(X)$ and $B_{m}(X), m \leq 10$ 
TABLE 2. Coefficients of $A_{5}(X) B_{5}(X)^{d}$ and of $F_{l, 5}(X)$

\begin{tabular}{|c|c|c|c|c|c|c|c|c|c|c|}
\hline$l:$ & 11 & 31 & 41 & 61 & 71 & 101 & 131 & 151 & 181 & 191 \\
\hline$d:$ & 2 & 6 & 8 & 12 & 14 & 20 & 26 & 30 & 36 & 38 \\
\hline$n: 0$ & 1 & 1 & 1 & 1 & 1 & 1 & 1 & 1 & 1 & 1 \\
\hline 1 & 1 & 1 & 1 & 1 & 1 & 1 & 1 & 1 & 1 & 1 \\
\hline 2 & 3 & 3 & 3 & 3 & 3 & 3 & 3 & 3 & 3 & 3 \\
\hline 3 & 0 & 11 & 11 & 11 & 11 & 11 & 11 & 11 & 11 & 11 \\
\hline 4 & 0 & 44 & 44 & -17 & 44 & 44 & 44 & 44 & 44 & 44 \\
\hline 5 & 0 & 36 & -53 & -169 & -156 & -300 & -444 & -540 & -684 & -732 \\
\hline 6 & 0 & 32 & 153 & 325 & -250 & 316 & 172 & 76 & -973 & -116 \\
\hline 7 & 0 & 0 & -160 & 167 & 749 & 1991 & 1660 & -893 & -870 & -2069 \\
\hline 8 & 0 & 0 & 59 & -804 & 1560 & -80 & -4713 & 5721 & 2782 & 2818 \\
\hline
\end{tabular}

in $l$ for $p<n \leq 2 p$, etc. For instance, if $m=7$ we have

$$
\begin{aligned}
& B_{7}(X)=1-720 X^{7}-48389400 X^{14}-\cdots, \\
& A_{7}(X)=1+X+4 X^{2}+20 X^{3}+110 X^{4}+638 X^{5}+3828 X^{6}+23412 X^{7}+\cdots,
\end{aligned}
$$

so the coefficient of $X^{5}$ in $F_{l, m}(X)$ is 638 for all but finitely many primes $l \equiv 1(\bmod 7) \quad($ in fact, as we shall see, for all such $l$ except $29,43,71,113$, $197,421,463$ and 547) and the coefficient of $X^{7}$ equals $(164604-720 l) / 7$ for all but finitely many $l$ (namely, those mentioned and 211, 239, 281, 337, $379,449,757,1583,1597$ and 2689). Table 2 illustrates Theorem 1 in the case $m=5$ by giving the first coefficients of $F_{l, 5}(X)$ for some small values of $l$ and showing (broken line) how long each polynomial $F_{l, 5}(X)$ agrees with the power series

$$
\begin{aligned}
A_{5}(X) B_{5}(X)^{d}= & +X+3 X^{2}+11 X^{3}+44 X^{4}+(180-24 d) X^{5} \\
& +(796-24 d) X^{6}+(3532-72 d) X^{7}+(15906-264 d) X^{8} \\
& +(72490-1056 d) X^{9}+\left(331282-15660 d+288 d^{2}\right) X^{10} \\
& +\left(1544418-30444 d+288 d^{2}\right) X^{11} \\
& +\left(7211960-118788 d+864 d^{2}\right) X^{12} \\
& +\left(33850952-506484 d+3168 d^{2}\right) X^{13} \\
& +\left(159612948-2238720 d+12672 d^{2}\right) X^{14}+\cdots \\
& \quad(l=5 d+1) .
\end{aligned}
$$

Clearly, the theorem is equivalent to saying that the $n$th coefficient of the logarithm of $F_{l, m}(X)$ is a linear function of $l$ for all but finitely many $l$ (for fixed $m$ and $n$ ). More precisely, write

$$
B_{m}(X)=\exp \left(-\sum_{n=1}^{\infty} \beta_{m}(n) \frac{X^{n}}{n}\right)
$$

(it turns out that $\beta_{m}(n)$ is integral and nonnegative, which is the reason for including the minus sign and the factor $1 / n)$; then Theorem 1, except for the integrality statement, is equivalent to

Theorem $1^{\prime}$. Fix integers $m, n \in \mathbb{N}$. Then the coefficient of $X^{n}$ in $\log F_{l, m}(X)$ equals

$$
\frac{m^{n}-l \beta_{m}(n)}{m n}
$$


for all $l \equiv 1(\bmod m)$ not belonging to an effectively determinable finite set $\mathscr{E}_{m}(n)$.

In the notation of this theorem, the set of exceptional primes in Theorem 1 is simply $\bigcup_{n<N} \mathscr{E}_{m}(n)$, and the "new" exceptional primes for given $n$ are the elements of the set $\mathscr{E}_{m}^{0}(n)=\mathscr{E}_{m}(n) \backslash \bigcup_{n^{\prime}<n} \mathscr{E}_{m}\left(n^{\prime}\right)$. Examples of the sets $\mathscr{E}_{m}^{0}(n)$ for $m \leq 12$ and some small values of $n$ are given in Table 3 (when this set contains 25 or more primes, we have given only its first three and last three elements and its cardinality). The way to determine these sets will be explained in $\S 3$.

In general, the coefficients $\beta_{m}(n)$, and hence the power series $B_{m}(X)$ and $A_{m}(X)$ occurring in Theorem 1, are difficult to determine. A simple description of these coefficients in certain special cases is given by the following theorem:

Theorem 2. The coefficients $\beta_{m}(n)$ for $m=p^{j}$ ( $p$ prime, $j \geq 1$ ) and $m=2 p$ $(p>2$ prime $)$ are given by the generating functions

$$
\begin{aligned}
& \sum_{n=0}^{\infty} \frac{\beta_{p^{j}}(n)}{n !} X^{n}=\left(\sum_{\nu=0}^{\infty} \frac{X^{p \nu}}{\nu ! p}\right)^{p^{j-1}}, \\
& \sum_{n=0}^{\infty} \frac{\beta_{2 p}(n)}{n !} X^{n}=\sum_{r=-\infty}^{\infty}\left(\sum_{\nu=0}^{\infty} \frac{X^{2 \nu+|r|}}{\nu !(\nu+|r|) !}\right)^{p} .
\end{aligned}
$$

\begin{tabular}{|c|c|c|c|c|}
\hline & $m=3$ & $m=4$ & $m=5$ & $m=6$ \\
\hline$n=3$ & - & 5 & 11 & 7 \\
\hline 4 & 7 & - & 61 & 13 \\
\hline 5 & 13 & 13,17 & 41 & 19 \\
\hline 6 & - & - & $31,71,181,521$ & 31 \\
\hline 7 & 19,31 & 29,37 & $101,151,191,461$ & 37,43 \\
\hline 8 & 43 & - & $131,241,251,401,421,991$ & - \\
\hline 9 & - & 41,53 & $281,331,491,641,881,941,1871$ & $61,67,73$ \\
\hline 10 & 37,73 & - & $271,311,661,1181$ & 79 \\
\hline 11 & 67 & $61,73,101$ & \begin{tabular}{|c|}
$211,431,601,631,691,751$ \\
$1051,1481,1531,1621,1741,2531$ \\
$2801,3001,3011,9091$
\end{tabular} & 97,103 \\
\hline 12 & - & - & $\begin{array}{c}701,761,971,1021,1201,1321 \\
1381,1511,1721,1801,2141,2371 \\
2441,2741,5051,13421\end{array}$ & 109 \\
\hline 13 & $61,79,103$ & $89,97,109$ & \begin{tabular}{|c|}
$541,571,811,821,1031,1171$ \\
$1291,1301,1471,1861,1901,2381$ \\
$2551,2671,4421,4561,4621,4831$ \\
$7741,7841,12391,19141$ \\
\end{tabular} & $127,139,157$ \\
\hline 14 & 97,157 & - & $\begin{array}{c}911,961,1061,1151,1451,1571 \\
2081,2251,2351,2411,2791,3121 \\
3301,3371,3511,3931,4001,4201 \\
4231,4441,6121,6521,6971\end{array}$ & 151,163 \\
\hline 15 & - & $113,137,173,197$ & $\begin{array}{c}1091,2011,2161,2221,3221 \\
3331,4951\end{array}$ & $181,199,211$ \\
\hline
\end{tabular}

TABLE 3. New exceptional primes for $m \leq 6$ 
TABLE 3 (continued). New exceptional primes for $7 \leq m \leq 12$

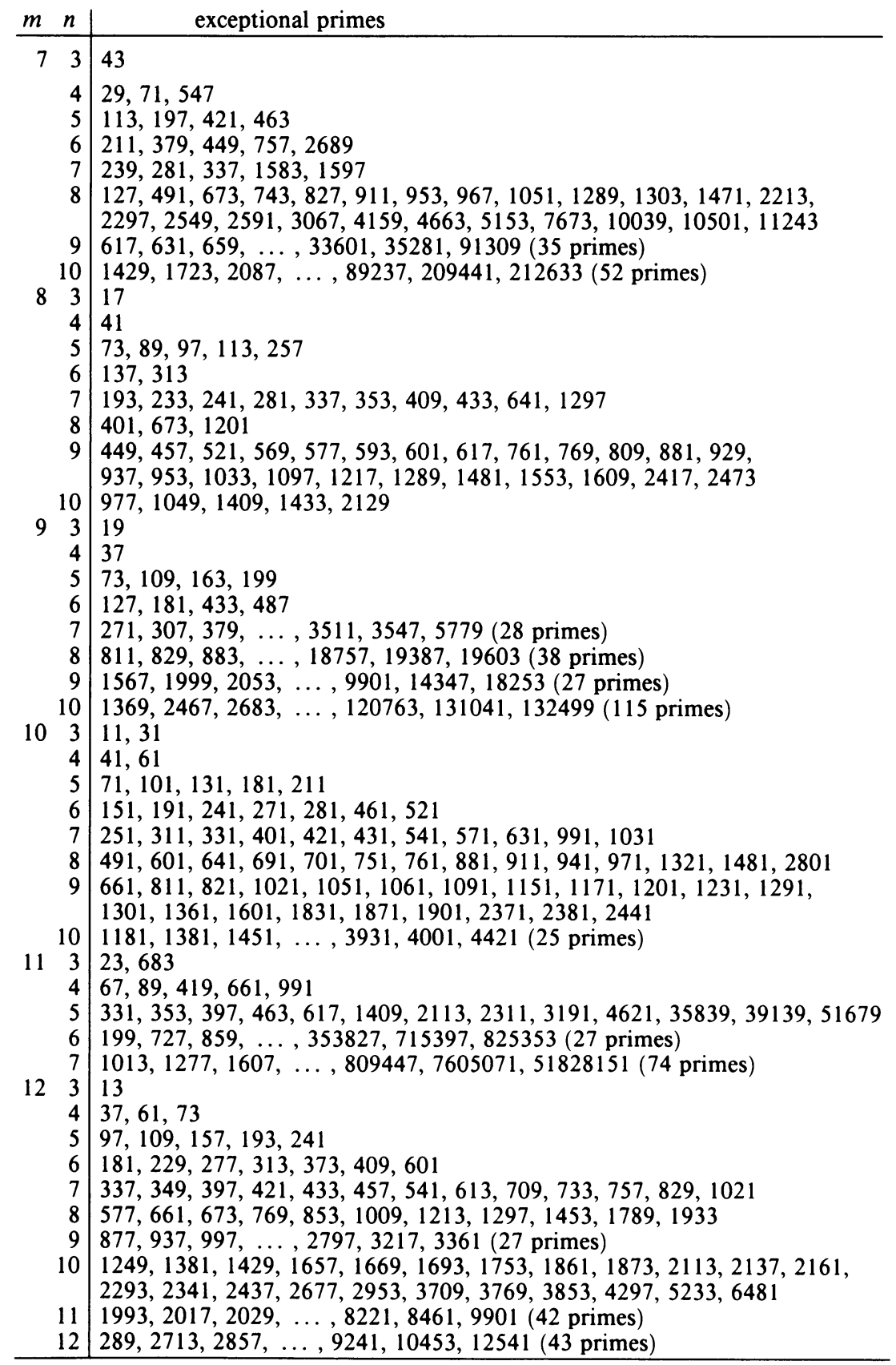

In particular, the coefficients $\beta_{m}(n)$ for $m=p$ ( $p$ prime) and $m=4$ are given by

$$
\beta_{p}(n)=\left\{\begin{array}{cl}
\frac{n !}{(n / p) ! p} & \text { if } n \equiv 0(\bmod p) \\
0 & \text { otherwise }
\end{array}\right.
$$




$$
\beta_{4}(n)= \begin{cases}\frac{n !^{2}}{(n / 2) !^{4}} & \text { if } n \equiv 0(\bmod 2), \\ 0 & \text { otherwise. }\end{cases}
$$

If $m=p^{j}$ ( $p$ prime, $\left.j \geq 1\right)$, then the coefficient $\beta_{m}(n)$ is nonzero only if $p$ divides $n$.

The cases $m=3$ and 4 of this theorem were proved by D. and E. Lehmer [3] and the case $m=p$ by S. Gurak [2] (Corollary 2, p. 322).

If $m=2$, then we can sum the series $\sum \beta_{2}(n) X^{n} / n$ explicitly to give closed formulas for the power series $A_{2}(X)$ and $B_{2}(X)$. However, here one can give much more information than in Theorem 1 , since $f_{l, 2}$ can be calculated in closed form, essentially as a Chebyshev polynomial (Theorem 3). The result, which will be proven in $\S 4$, is the formula

$$
f_{l, 2}(X)=\sum_{n=0}^{d}(-1)^{\left[\frac{d-n}{2}\right]}\left(\begin{array}{c}
{\left[\frac{d+n}{2}\right]} \\
n
\end{array}\right) X^{n} \quad\left(d=\frac{l-1}{2}\right) .
$$

It was essentially known to Gauss (Disquisitiones Arithmeticae, Article 337).

As mentioned in the beginning of the section, several of our results overlap with the results in the papers [1] and [2] by S. Gurak, whose existence we learned about after completing our work. Specifically, Gurak proves in [1] that the coefficient of $X^{n}$ in $F_{l, m}(X)$ is $P_{m, n}(l)$ for primes $l>n^{\phi(m)} \quad(\phi=$ Euler totient function), where $P_{m, n}$ is a polynomial of degree $\leq n / p \quad(p=$ smallest prime factor of $m$ ). Equation (10) of [1] (resp. equation (28) of [2]) is equivalent to our Theorem $1^{\prime}$ and thus to all parts of Theorem 1 except for the integrality of the power series $A_{m}$ and $B_{m}$. The explicit description of these power series in the special cases $m=p$ and $m=4$ were also given in [2], as mentioned after Theorem 2. The description of the algorithm for finding the exceptional sets $\mathscr{E}_{m}(n)$, and the numerical computations concerning them, are new.

\section{Proof of Theorem 1}

Using the Taylor series of $\log (1-T)$ around $T=0$, we find

$$
\log F_{l, m}(X)=-\sum_{n=1}^{\infty}\left(\sum_{r \in \mathscr{R}}\left(\zeta^{r}+\zeta^{\lambda r}+\zeta^{\lambda^{2} r}+\cdots+\zeta^{\lambda^{m-1} r}\right)^{n}\right) \frac{X^{n}}{n} .
$$

Replacing the inner sum by one over all $r \in(\mathbb{Z} / l \mathbb{Z})^{\times}$simply multiplies it by $m$, since the value of the summand is independent of the choice of coset representative $r$, and there are $m$ cosets. Hence, the inner sum equals

$$
\begin{aligned}
\frac{1}{m} \sum_{r \neq 0(\bmod l)}\left(\sum_{i=0}^{m-1} \zeta^{\lambda^{i} r}\right)^{n} & =-m^{n-1}+\frac{1}{m} \sum_{r(\bmod l)} \sum_{i_{1}, \ldots, i_{n}=0}^{m-1} \zeta^{\left(\lambda^{i_{1}}+\cdots \lambda^{i_{n}}\right) r} \\
& =-m^{n-1}+\frac{l}{m} \beta_{l, m}(n),
\end{aligned}
$$

where

$$
\beta_{l, m}(n)=\#\left\{\left(i_{1}, \ldots, i_{n}\right) \in(\mathbb{Z} / m \mathbb{Z})^{n} \mid \lambda^{i_{1}}+\cdots+\lambda^{i_{n}}=0 \text { in } \mathbb{Z} / l \mathbb{Z}\right\}
$$

(recall that $\lambda$ here refers to a fixed primitive $m$ th root of unity in $(\mathbb{Z} / l \mathbb{Z})^{\times}$); 
to obtain this formula, we have added and subtracted a term corresponding to $r=0$ and then used the fact that $\sum_{r(\bmod l)} \zeta^{k r}$ equals $l$ if $k \equiv 0(\bmod l)$ and vanishes otherwise.

We now define coefficients $\beta_{m}(n)(n \geq 1)$ by

$$
\beta_{m}(n)=\#\left\{\left(i_{1}, \ldots, i_{n}\right) \in(\mathbb{Z} / m \mathbb{Z})^{n} \mid \lambda_{0}^{i_{1}}+\cdots+\lambda_{0}^{i_{n}}=0\right\},
$$

where $\lambda_{0}=e^{2 \pi i / m}$ is a primitive root of unity in the complex number field rather than in the finite field $\mathbb{F}_{l}$. We will show that $\beta_{l, m}(n)=\beta_{m}(n)$ for all but finitely many primes $l \equiv 1(\bmod m)$, for fixed $m$ and $n$. This implies Theorem $1^{\prime}$ and hence, with $B_{m}(X)$ defined by equation (3), Theorem 1 (except for the asserted integrality of the power series $A_{m}$ and $\left.B_{m}\right)$, because for $l=m d+1$ prime and sufficiently large (depending on $N$ and $m$ ) we have

$$
\begin{aligned}
\log F_{l, m}(X) & \equiv \sum_{n=1}^{N-1}\left(\frac{m^{n}}{m}-\frac{m d+1}{m} \beta_{m}(n)\right) \frac{X^{n}}{n} \\
& \equiv-\frac{1}{m} \log (1-m X)+\left(d+\frac{1}{m}\right) \log B_{m}(X) \\
& \equiv \log A_{m}(X)+d \log B_{m}(X) \quad\left(\bmod X^{N}\right)
\end{aligned}
$$

Let $\Phi_{m}(x)$ be the $m$ th cyclotomic polynomial, i.e., the irreducible monic polynomial (of degree $\varphi(m)$, the Euler totient function of $m$ ) of $\lambda_{0}$ over $\mathbb{Q}$. Then a polynomial $P(x)$ with rational coefficients vanishes at $\lambda_{0}$ if and only if it is divisible by $\Phi_{m}(x)$. Thus,

$$
\beta_{m}(n)=\#\left\{\mathbf{i}=\left(i_{1}, \ldots, i_{n}\right) \in(\mathbb{Z} / m \mathbb{Z})^{n}\left|\Phi_{m}(x)\right| P_{\mathbf{i}}(x)\right\},
$$

where $P_{\mathbf{i}}(x)$ denotes the polynomial $x^{i_{1}}+\cdots x^{i_{n}}$. (Strictly speaking, this is not a polynomial unless we choose actual integer representatives of the classes $i_{\nu}$ $(\bmod m)$, say $0 \leq i_{\nu}<m$, but obviously the condition that $\Phi_{m}$ divide $P_{\mathbf{i}}$ is independent of this choice, since $\Phi_{m}(x) \mid\left(x^{m}-1\right)$.) Obviously, every $n$-tuple i contributing to $\beta_{m}(n)$ also contributes to $\beta_{l, m}(n)$, since $\lambda \in \mathbb{F}_{l}^{\times}$is a root of the polynomial $\Phi_{m}(x)$ and hence of all of its multiples. Hence, $\beta_{l, m}(n)$ is at least as large as $\beta_{m}(n)$, but might be larger. However, the fact that $\Phi_{m}(x)$ is irreducible over $\mathbb{Q}$ means that the g.c.d. of $\Phi_{m}(x)$ with any polynomial $P(x)$ not divisible by it is 1 . In particular, if $\mathbf{i}$ is an index in $(\mathbb{Z} / m \mathbb{Z})^{n}$ not counted in $\beta_{m}(n)$, i.e., one for which $P_{\mathrm{i}}$ is not divisible by $\Phi_{m}$, then there are polynomials with rational coefficients $g(x)$ and $h(x)$ (depending on $m$ and $n)$ such that $g(x) \Phi_{m}(x)+h(x) P_{\mathbf{i}}(x) \equiv 1$. If the prime $l \equiv 1(\bmod m)$ does not occur in the denominator of any coefficient of $g$ or $h$, then we can reduce this equation $(\bmod l)$, and the fact that $\Phi_{m}(\lambda)$ vanishes then implies that $P_{\mathbf{i}}(\lambda)$ is also nonzero modulo $l$. The condition that $l$ does not occur in the denominators of $g$ or $h$ is equivalent to the condition that $l$ should not divide the resultant of $\Phi_{m}$ and $P_{\mathrm{i}}$ (essentially, the smallest positive integer $R$ such that the equation $g(x) \Phi_{m}(x)+h(x) P_{\mathrm{i}}(x) \equiv R$ is solvable with polynomials $g, h \in \mathbb{Z}[x])$. Therefore, Theorem $1^{\prime}$ is true if we take for $\mathscr{E}_{m}(n)$ the union over all $\mathbf{i} \in(\mathbb{Z} / m \mathbb{Z})^{n}$ with $\Phi_{m} \nmid P_{\mathbf{i}}$ of the set of primes $l \equiv 1(\bmod m)$ dividing the resultant of $\Phi_{m}$ and $P_{\mathbf{i}}$. 
It remains to prove the integrality of the coefficients of the power series $A_{m}(X)$ and $B_{m}(X)$. Pick an integer $N>0$. By Theorem 1 , there is an integer $d_{0}$ (depending on $m$ and $N$ ) such that the first $N$ coefficients of $A_{m} B_{m}^{d}$ are equal to those of $F_{l, m}$, and hence integral, for all $d \geq d_{0}$ for which $l=m d+1$ is prime. If $d_{1}$ and $d_{2}$ are two such values of $d$, then it follows by division (since the power series $A_{m}$ and $B_{m}$ start with 1) that $B_{m}^{d_{1}-d_{2}}$ also has integral coefficients up to degree $N$. But if $B_{m}^{a}$ and $B_{m}^{b}$ have integral coefficients, then so does $B_{m}^{(a, b)}$, where $(a, b)$ denotes the g.c.d. of $a$ and $b$, because this g.c.d. can be written as $a r+b s$ for some integers $r$ and $s$. This proves that $B_{m}(X)^{\mu}$ has integral coefficients up to order $N$, where $\mu$ is the greatest common divisor of all of the differences $d_{1}-d_{2}$ with $d_{i} \geq d_{0}$ and $m d_{i}+1$ prime. Dirichlet's theorem on primes in arithmetic progression implies that $\mu$ equals 1 if $m$ is even and 2 if $m$ is odd. (Indeed, it is obvious that this value of $\mu$ divides all $d$, and hence all differences of $d$, with the stated property. Conversely, the definition of $\mu$ says that all sufficiently large primes $l$ which are congruent to 1 modulo $m$ are in fact congruent to a fixed number $l_{1}$ modulo $\mu \mathrm{m}$, and hence-by Dirichlet's theorem-that there is only one arithmetic progression $\{k \mu m+c\}_{k \geq 1}$ with $(c, \mu m)=1$ and $c \equiv 1(\bmod m)$. But this implies that $\varphi(\mu m)=\varphi(m)$ and hence that $\mu=1$ if $m$ is even, $\mu \mid 2$ if $m$ is odd.) Therefore, $B_{m}^{d}$ has integral coefficients up to degree $N$ for large $d$ with $m d+1$ prime, so the fact that $A_{m} B_{m}^{d}$ also has integral coefficients up to this degree implies that $A_{m}$ does as well. Since $N$ was arbitrary, it follows that $A_{m}(X)$ has integral coefficients, as claimed, and then $B_{m}(X)$ must, too, because of equation (1). (For $m$ odd, the first part of our argument gave only the integrality of the coefficients of the square of $B_{m}$.) This completes the proof of Theorem 1.

Remark. For $m \geq 1$ define

$$
C_{m}(X)=\exp \left(\sum_{n=1}^{\infty} \frac{(n m-1) !}{n !^{m}} X^{n}\right)
$$

Then Theorem 2 implies that $C_{m}(X)$ has integral coefficients when $m$ is prime (since then $\left.C_{m}(X)=B_{m}\left(X^{1 / m}\right)^{-1}\right)$. Numerical calculations for small $m$ suggested that this remains true also for $m$ composite. Maxim Kontsevich showed us how to prove the stronger assertion that $C_{m}(X)^{1 /(m-1) !}$ has integral coefficients. This is best possible, since $C_{m}(X)$ begins with $1+(m-1) ! X+O\left(X^{2}\right)$.

\section{Proof of Theorem 2 and Determination of the exceptional SeT}

In this section we discuss the computation of the coefficients $\beta_{m}(n)$ and $\beta_{l, m}(n)$ and the determination of the primes $l$ for which they differ.

Obviously, the polynomial $P_{\mathbf{i}}(x)$ corresponding to $\mathbf{i}=\left(i_{1}, \ldots, i_{n}\right)$ depends only on the set $\mathbf{i}$ up to permutation and hence on the numbers $n_{i}=\#\left\{\nu \mid i_{\nu}=\right.$ $i\}, 0 \leq i \leq m-1$. These numbers $n_{i}$ satisfy $n_{i} \geq 0$ and $n_{0}+\cdots+n_{m-1}=n$; conversely, for any such integers $n_{i}$ there are $n ! /\left(n_{0} ! \cdots n_{m-1} !\right)$ corresponding $n$-tuples $\mathbf{i}$, for each of which $P_{\mathbf{i}}(x)$ is the polynomial $n_{0}+n_{1} x+\cdots+n_{m-1} x^{m-1}$. Hence, 


$$
\beta_{m}(n)=\sum_{\substack{n_{0}, n_{1}, \ldots, n_{m-1} \geq 0 \\ n_{0}+n_{1}+\cdots+n_{m-1}=n \\ \Phi_{m}(x) \mid n_{0}+n_{1} x+\cdots+n_{m-1} x^{m-1}}} \frac{n !}{n_{0} ! \cdots n_{m-1} !} .
$$

The third condition in the summation can be replaced by $n_{0}+n_{1} \lambda_{0}+\cdots+$ $n_{m-1} \lambda_{0}^{m-1}=0$, and if $\lambda_{0}$ is replaced in this with the $m$ th root of unity $\lambda \in$ $\mathbb{Z} / l \mathbb{Z}$, then the same expression gives a formula for $\beta_{l, m}(n)$.

We now consider $m$ of some special types. If $m=p$ is prime, then the cyclotomic polynomial $\Phi_{m}$ is the polynomial $1+x+\cdots+x^{p-1}$ of degree $m-1$, so the last condition under the summation sign in (8) can be satisfied only if $n_{0}=n_{1}=\cdots=n_{m-1}$, in which case the common value must be $n / m=n / p$. This proves (6) of Theorem 2. More generally, if $m=p^{j}$, then $\Phi_{m}(x)=$ $\left(x^{m}-1\right) / x^{m^{\prime}-1}=1+x^{m^{\prime}}+\cdots+x^{m^{\prime}(p-1)}$, where $m^{\prime}=m / p=p^{j-1}$. If this divides $n_{0}+n_{1} x+\cdots+n_{m-1} x^{m-1}$, then the quotient is $n_{0}+n_{1} x+\cdots+n_{m^{\prime}-1} x^{m^{\prime}-1}$ and the sequence $\left(n_{0}, n_{1}, \ldots, n_{m-1}\right)$ is just the sequence $\left(n_{0}, n_{1}, \ldots, n_{m^{\prime}-1}\right)$ repeated $p$ times. Hence,

$$
\beta_{m}(n)=\sum_{\substack{n_{0}, n_{1}, \ldots, n_{m^{\prime}-1} \geq 0 \\ p\left(n_{0}+n_{1}+\cdots+n_{m^{\prime}-1}\right)=n}} \frac{n !}{\left(n_{0} ! \cdots n_{m^{\prime}-1} !\right)^{p}} .
$$

Multiplying this by $X^{n} / n$ ! and summing over $n$, we obtain the generating function given in (4). Obviously, (6) is a special case of (4), but we have preferred to give it separately since it is simpler and since here one can give a closed formula for each $\beta_{m}(n)$. This is also the case for $m=4$, where equation (4) gives $\beta_{4}(n)=0$ for $n$ odd and

$$
\beta_{4}(2 n)=\sum_{n_{0}+n_{1}=n} \frac{(2 n) !}{n_{0} !^{2} n_{1} !^{2}}=\left(\begin{array}{c}
2 n \\
n
\end{array}\right) \sum_{n_{0}=0}^{n}\left(\begin{array}{c}
n \\
n_{0}
\end{array}\right)^{2}=\left(\begin{array}{c}
2 n \\
n
\end{array}\right)^{2}
$$

proving equation (7). Finally, if $m=2 p$ with $p$ an odd prime, then $\Phi_{m}(x)=$ $\left(x^{p}+1\right) /(x+1)=1-x+x^{2}-\cdots+x^{p-1}$, and it is easily checked that $n_{0}+$ $n_{1} x+\cdots+n_{2 p-1} x^{2 p-1}$ is divisible by this if and only if $\left(n_{0}, n_{1}, \ldots, n_{2 p-1}\right)$ has the form $\left(n_{0}, n_{1}, \ldots, n_{p-1}, n_{0}+r, n_{1}-r, \ldots, n_{p-1}+r\right)$ for some integers $n_{0}, \ldots, n_{p-1}$ and $r$ satisfying $2\left(n_{0}+\cdots+n_{p-1}\right)+r=n$; this easily leads to (5) by setting $A=n_{0}+\cdots+n_{p-1}$ if $r \geq 0, A=n_{0}+\cdots+n_{p-1}+r$ if $r<0$. In general, the smaller the difference between $m-1$ and $\varphi(m)$ the easier it is to analyze the divisibility condition in (8) and hence to give an explicit description of the coefficients $\beta_{m}(n)$.

Finally, we discuss the computation of the exceptional set $\mathscr{E}_{m}(n)$. For fixed values of $m$ and $n$, we consider $n$-tuples $\mathbf{i}=\left(i_{1}, \ldots, i_{n}\right) \in(\mathbb{Z} / m \mathbb{Z})^{n}$ and denote by $R_{\mathrm{i}}$ the resultant of the polynomials $P_{\mathrm{i}}$ and $\Phi_{m}(x)$. This resultant is easily computed as the norm of the algebraic number $P_{\mathbf{i}}\left(\lambda_{0}\right)$, i.e., as the product of the numbers $P_{\mathrm{i}}(\lambda)$ as $\lambda$ ranges over the primitive $m$ th roots of unity in $\mathbb{C}$. The number of $n$-tuples $\mathbf{i}$ with $R_{\mathbf{i}}=0$ is $\beta_{m}(n)$, and the primes $l \equiv 0(\bmod m)$ dividing any nonzero $R_{\mathrm{i}}$ are the members of the exceptional set $\mathscr{E}_{m}(n)$. Obviously two values of $\mathbf{i}$ which are equivalent under (i) permutation of the $i_{j}$ 's, (ii) translations $i_{j} \mapsto i_{j}+c(\bmod m)$ for any $c \in(\mathbb{Z} / m \mathbb{Z})$, or (iii) scalings $i_{j} \mapsto k i_{j}(\bmod m)$ for any $k \in(\mathbb{Z} / m \mathbb{Z})^{\times}$give the same value of $R_{\mathbf{i}}$, 
TABLE 4. Examples of resultant computations

\begin{tabular}{cc}
$m=7$, & $n=5:$ \\
$\mathbf{i}$ & $R_{\mathbf{i}}$ \\
\hline$(0,0,0,0,1)$ & $29 \cdot 113$ \\
$(0,0,0,1,1)$ & 463 \\
$(0,0,0,1,2)$ & 421 \\
$(0,0,0,1,3)$ & $2^{3} \cdot 71$ \\
$(0,0,0,1,6)$ & $13^{2}$ \\
$(0,0,1,1,2)$ & 29 \\
$0,0,1,1,3)$ & 197 \\
$(0,0,1,1,4)$ & 1 \\
$(0,0,1,2,3)$ & 43 \\
$(0,0,1,2,4)$ & $2^{6}$ \\
$(0,0,1,2,5)$ & 29 \\
$(0,0,1,2,6)$ & $2^{3}$ \\
$(0,1,2,3,4)$ & 1
\end{tabular}

\begin{tabular}{cc}
$m=11$, & $n=4:$ \\
$\mathbf{i}$ & $R_{\mathbf{i}}$ \\
\hline$(0,0,0,1)$ & $67 \cdot 661$ \\
$(0,0,1,1)$ & $2{ }^{10}$ \\
$(0,0,1,2)$ & $23^{2}$ \\
$(0,0,1,3)$ & 991 \\
$(0,0,1,5)$ & $23 \cdot 67$ \\
$(0,0,1,7)$ & 419 \\
$(0,0,1,10)$ & 1 \\
$(0,1,2,3)$ & 1 \\
$(0,1,2,4)$ & 23 \\
$(0,1,2,5)$ & 89 \\
$(0,1,3,4)$ & 1
\end{tabular}

$m=12, n=3$ :

\begin{tabular}{cc}
$\mathbf{i}$ & $\boldsymbol{R}_{\mathbf{i}}$ \\
\hline$(0,0,1)$ & 13 \\
$(0,0,2)$ & $7^{2}$ \\
$(0,0,3)$ & $5^{2}$ \\
$(0,0,4)$ & $3^{2}$ \\
$(0,0,6)$ & 1 \\
$(0,1,2)$ & $2^{2}$ \\
$0,1,3)$ & 13 \\
$0,1,4)$ & 1 \\
$(0,1,5)$ & $2^{2}$ \\
$(0,1,6)$ & 1 \\
$(0,2,4)$ & $2^{4}$ \\
$(0,2,6)$ & 1 \\
$(0,3,6)$ & 1
\end{tabular}

so we need only compute the resultants for inequivalent values of $i$. Table 4 shows the results of this computation for all inequivalent $n$-tuples $\mathbf{i}$ in the cases $(m, n)=(7,5),(11,4)$, and $(12,3)$, illustrating the way that the corresponding entries of Table 3 were computed. (Note that only the prime divisors of $R_{\mathbf{i}}$ congruent to 1 modulo $m$ must be taken-this is automatic if the prime occurs to the first power-and also that the primes occurring for a given value of $n$ may be new exceptional primes for any $n^{\prime} \leq n$.) However, in these three examples there are only about a dozen inequivalent $n$-tuples $\mathbf{i}$, whereas, for example, computing the final entry in Table 3 required looking at over thirty thousand inequivalent orbits.

\section{THE CASE $m=2$}

In this case we find from (3) and (6)

$$
\frac{B_{2}^{\prime}(X)}{B_{2}(X)}=-\sum_{n=1}^{\infty} \frac{(2 n) !}{n !^{2}} X^{2 n-1}=\frac{1}{X}\left(1-\frac{1}{\sqrt{1-4 X^{2}}}\right)
$$

from which we obtain the closed formulas and power series expansions

$$
\begin{aligned}
& B_{2}(X)=\frac{1}{2}\left(1+\sqrt{1-4 X^{2}}\right)=1-\sum_{n=0}^{\infty} \frac{\left(\begin{array}{c}
2 n \\
n
\end{array}\right)}{n+1} X^{2 n+2} \\
& A_{2}(X)=\frac{1}{2}\left(1+\sqrt{\frac{1+2 X}{1-2 X}}\right)=\frac{1}{2}+\sum_{n=0}^{\infty}\left(\begin{array}{c}
2 n \\
n
\end{array}\right)\left(\frac{1}{2} X^{2 n}+X^{2 n+1}\right) .
\end{aligned}
$$

However, here we can give complete formulas on the polynomials $F_{l, m}(X)$. First of all, the $m$ th root of unity $\lambda$ here is just $-1(\bmod l)$, so we can extend the definition of $F_{l, m}(X)$ to all odd values of $l$ by

$$
F_{l, 2}(X)=P_{d}(X):=\prod_{j=1}^{d}\left(1-\left(\zeta_{l}^{j}+\zeta_{l}^{-j}\right) X\right) \quad\left(\zeta_{l}=e^{2 \pi i / l}, \quad l=2 d+1\right)
$$


TABLE 5. Coefficients of $A_{2}(X) B_{2}(X)^{d}$

\begin{tabular}{|c|c|c|c|c|c|c|c|c|c|c|c|c|c|}
\hline & 1 & $X$ & $X^{2}$ & $X^{3}$ & $X^{4}$ & $X^{5}$ & $X^{6}$ & $X^{7}$ & $X^{8}$ & $X^{9}$ & $X^{10}$ & $X^{11}$ & \\
\hline$d=1$ & 1 & 1 & 0 & 1 & 1 & 3 & 4 & 10 & 15 & 35 & 56 & 126 & $\ldots$ \\
\hline 2 & 1 & 1 & -1 & 0 & 0 & 1 & 1 & 4 & 5 & 15 & 21 & 56 & . \\
\hline 3 & 1 & 1 & -2 & -1 & 0 & 0 & 0 & 1 & 1 & 5 & 6 & 21 & $\ldots$ \\
\hline 4 & 1 & 1 & -3 & -2 & 1 & 0 & 0 & 0 & 0 & 1 & 1 & 6 & $\ldots$ \\
\hline 5 & 1 & 1 & -4 & -3 & 3 & 1 & 0 & 0 & 0 & 0 & 0 & 1 & $\ldots$ \\
\hline 6 & 1 & 1 & -5 & -4 & 6 & 3 & -1 & 0 & 0 & 0 & 0 & 0 & $\cdots$ \\
\hline 7 & 1 & 1 & -6 & -5 & 10 & 6 & -4 & -1 & 0 & 0 & 0 & 0 & $\ldots$ \\
\hline
\end{tabular}

Theorem 3. The polynomials $P_{d}(X)$ are given by the closed formula

$$
\begin{aligned}
P_{d}(X)= & \frac{1}{2}\left(1+\sqrt{\frac{1+2 X}{1-2 X}}\right)\left(\frac{1+\sqrt{1-4 X^{2}}}{2}\right)^{d} \\
& +\frac{1}{2}\left(1-\sqrt{\frac{1+2 X}{1-2 X}}\right)\left(\frac{1-\sqrt{1-4 X^{2}}}{2}\right)^{d},
\end{aligned}
$$

by the recursion

$$
P_{d}(X)=P_{d-1}(X)-X^{2} P_{d-2}(X) \quad(d \geq 2)
$$

(with the initial conditions $P_{0}(X)=1, P_{1}(X)=1+X$ ), by the generating function

$$
\sum_{d=0}^{\infty} P_{d}(X) T^{d}=\frac{1+X T}{1-T+X^{2} T^{2}}
$$

or by the expansion

$$
P_{d}(X)=\sum_{n=0}^{d}(-1)^{[n / 2]}\left(\begin{array}{c}
{\left[d-\frac{n}{2}\right]} \\
{\left[\frac{n}{2}\right]}
\end{array}\right) X^{n} .
$$

Clearly, this sharpens Theorem 1: equation (9) implies that the congruence $F_{l, 2}=A_{2} B_{2}^{d}$ holds modulo $X^{2 d+1}$, which determines $F_{l, 2}$ completely since its degree equals $d$ (cf. Table 5), while (12) gives all the coefficients of the polynomial $F_{l, 2}(X)$ explicitly.

Proof. Define $P_{d}$ by (9) (rather than as $F_{l, 2}$ ). Then

$$
\begin{aligned}
\sum_{d=0}^{\infty} P_{d}(X) T^{d} & =\frac{\frac{1}{2}\left(1+\sqrt{\frac{1+2 X}{1-2 X}}\right)}{1-\frac{1}{2}\left(1+\sqrt{1-4 X^{2}}\right) T}+\frac{\frac{1}{2}\left(1-\sqrt{\frac{1+2 X}{1-2 X}}\right)}{1-\frac{1}{2}\left(1-\sqrt{1-4 X^{2}}\right) T} \\
& =\frac{1+X T}{1-T+X^{2} T^{2}} .
\end{aligned}
$$

This proves (11). The recursion (10) follows by multiplying both sides by $1-$ $T+X^{2} T^{2}$ and comparing coefficients of $T^{d}$, while (12) follows by expanding the right-hand side as a geometric series

$$
\frac{1+T X}{1-T+T^{2} X^{2}}=(1+T X) \sum_{n=0}^{\infty} \frac{(-1)^{n} T^{2 n} X^{2 n}}{(1-T)^{2 n+1}}=\sum_{n=0}^{\infty} \frac{(-1)^{[n / 2]} T^{n}}{(1-T)^{2[n / 2]+1}} X^{n}
$$

and using the binomial theorem. This proves the equivalence of (9)-(12). 
Now let $Q_{d}(X)=X^{d} P_{d}\left(X^{-1}\right)$, the reciprocal polynomial of $P_{d}(X)$. Then the generating function of the $Q_{d}(X)$ is

$$
\sum_{d=0}^{\infty} Q_{d}(X) T^{d}=\sum_{d=0}^{\infty} P_{d}\left(X^{-1}\right)(X T)^{d}=\frac{1+T}{1-X T+T^{2}}
$$

Substituting $X=z+z^{-1}$ gives

$$
\begin{aligned}
\sum_{d=0}^{\infty} Q_{d}\left(z+z^{-1}\right) T^{d} & =\frac{1+T}{1-\left(z+z^{-1}\right) T+T^{2}}=\frac{1+T}{(1-z T)\left(1-z^{-1} T\right)} \\
& =\frac{1}{1-z}\left(\frac{1}{1-z^{-1} T}-\frac{z}{1-z T}\right)=\sum_{d=0}^{\infty} \frac{z^{-d}-z^{d+1}}{1-z} T^{d}
\end{aligned}
$$

whence $Q_{d}\left(z+z^{-1}\right)=z^{-d}\left(1+z+z^{2}+\cdots+z^{2 d}\right)$. Thus $Q_{d}$ has the $d$ roots $\zeta_{l}^{j}+\zeta_{l}^{-j}(1 \leq j \leq d)$, where $l=2 d+1$. Since $Q_{d}$ is monic (because $P_{d}(X)$ has constant term 1) and has degree $d$, this property uniquely characterizes $Q_{d}(X)$, so $Q_{d}(X)=\prod_{j}\left(X-\zeta_{l}^{j}-\zeta_{l}^{-j}\right)$, the reciprocal polynomial of $F_{l, 2}$. This completes the proof of Theorem 3. We observe that the property of $Q_{d}$ just used can be rewritten via the substitution $z=e^{i \theta}$ as

$$
Q_{d}(2 \cos \theta)=\frac{\sin \left(d+\frac{1}{2}\right) \theta}{\sin \frac{1}{2} \theta}=\frac{\sin (d+1) \theta+\sin d \theta}{\sin \theta}
$$

so that $Q_{d}(X)$ can be expressed in terms of the classical Chebyshev polynomials $S_{n}(X)$ by $Q_{d}(X)=S_{2 d}(\sqrt{X})=S_{d}(X)+S_{d-1}(X)$.

\section{Composite $l$}

In this section we give a partial generalization of our results to the case of composite $l$. Let $m_{1}, \ldots, m_{s}$ be fixed positive integers and consider integers of the form $l=l_{1} \cdots l_{s}$, where the $l_{i}$ are distinct primes of the form $l_{i}=m_{i} d_{i}+1, d_{i}>1$. Then the group $G=(\mathbb{Z} / l \mathbb{Z})^{\times}$contains a subgroup $H$ of order $m=m_{1} \cdots m_{s}$ given by

$$
H=\left\{x(\bmod l) \mid x^{m_{i}} \equiv 1 \mathrm{cf} .\left(\bmod l_{i} \text { for } i=1, \ldots, s\right\} .\right.
$$

If we identify $G$ with the Galois group of $\mathbb{Q}(\zeta) / \mathbb{Q}(\zeta$ a primitive $l$ th root of unity), then $H$ corresponds to a subfield $K$ of $\mathbb{Q}(\zeta)$ of degree $d=d_{1} \cdots d_{s}$. This field contains, and by the result in the Appendix of [2] is in fact generated by, the number

$$
\omega=\operatorname{Tr}_{\mathbb{Q}(\zeta) / K}(\zeta)=\sum_{x \in H} \zeta^{x}
$$

We denote the conjugates $\sum_{x \in H} \zeta^{j x}(j \in G / H)$ of $\omega$ by $\omega^{(j)}$ and set

$$
F(X)=F_{l_{1}, \ldots, l_{s} ; m_{1}, \ldots, m_{s}}(X)=\prod_{j \in G / H}\left(1-\omega^{(j)} X\right) .
$$

Then the same calculation as at the beginning of $\S 2$ gives

$$
\log F(X)=-\sum_{n \geq 1} S_{n} X^{n} / n
$$


with

$$
\begin{aligned}
S_{n} & =\sum_{j \in G / H}\left(\omega^{(j)}\right)^{n}=\frac{1}{|H|} \sum_{j \in G} \sum_{x_{1}, \ldots, x_{n} \in H} \zeta^{j\left(x_{1}+\cdots+x_{n}\right)} \\
& =\frac{1}{m} \sum_{k \mid l} \mu\left(\frac{l}{k}\right) \cdot k \cdot \#\left\{\left(x_{1}, \ldots, x_{n}\right) \in H^{n} \mid x_{1}+\cdots+x_{n} \equiv 0(\bmod k)\right\},
\end{aligned}
$$

where the last equality follows from the standard evaluation of the Ramanujan $\operatorname{sum} \sum_{j \in G} \zeta^{j x}$. We can write each divisor $k$ of $l$ as $\prod_{i \in I} l_{i}$ for some subset $I$ of $J=\{1, \ldots, s\}$. The expression $\#\{\cdots\}$ is multiplicative in the obvious sense, and we find

$$
S_{n}=\frac{1}{m} \sum_{I \subset J} \prod_{i \notin I}\left(-m_{i}^{n}\right) \prod_{i \in I}\left(l_{i} \beta_{l_{i}, m_{i}}(n)\right) .
$$

Thus, if we define for any set $\mathscr{M}$ of (not necessarily distinct) positive integers a power series $B_{\mathscr{M}}(X)$ by

$$
B_{\mathscr{M}}(X)=\exp \left(-\sum_{n=1}^{\infty} \beta_{\mathscr{M}}(n) \frac{X^{n}}{n}\right), \quad \beta_{\mathscr{M}}(n):=\prod_{m \in \mathscr{M}} \beta_{m}(n)
$$

(this is a priori in $\mathbb{Q}[[X]]$ but actually in $\mathbb{Z}[[X]]$, as we shall see below), then we find that

$$
F(X) \equiv \prod_{I \subset J} B_{\mathscr{M}_{I}}\left(m_{I^{\prime}} X\right)^{(-1)^{s-|I|} \prod_{i \in I} l_{i} / m}\left(\bmod X^{N}\right)
$$

(with $\mathscr{M}_{I}=\left\{m_{i}, i \in I\right\}, m_{I^{\prime}}=\prod_{i \notin I} m_{i}$ ) for any $N>0$, so long as no $l_{i}$ is in the exceptional set $\mathscr{E}_{m_{i}}(n)$ for any $n<N$. For $s=1$ the power series on the right of (13) is $B_{\{m\}}(X)^{l / m} B_{\varnothing}(m X)^{-1 / m}$, which agrees with Theorem 1 since $B_{\{m\}}(X)=B_{m}(X)$ and $B_{\varnothing}(X)=1-X$. For $s=2$ we obtain instead

$$
\left(\frac{B_{\left\{m_{1}, m_{2}\right\}}(X)^{l_{1} l_{2}}\left(1-m_{1} m_{2} X\right)}{B_{m_{1}}\left(m_{2} X\right)^{l_{1}} B_{m_{2}}\left(m_{1} X\right)^{l_{2}}}\right)^{1 / m}
$$

or

$$
\begin{aligned}
& {\left[B_{\left\{m_{1}, m_{2}\right\}}(X)\right]^{d_{1} d_{2}}\left[\left(\frac{B_{\left\{m_{1}, m_{2}\right\}}(X)}{B_{m_{1}}\left(m_{2} X\right)}\right)^{1 / m_{2}}\right]^{d_{1}}\left[\left(\frac{B_{\left\{m_{1}, m_{2}\right\}}(X)}{B_{m_{2}}\left(m_{1} X\right)}\right)^{1 / m_{1}}\right]^{d_{2}}} \\
& \times\left[\left(\frac{B_{\left\{m_{1}, m_{2}\right\}}(X)\left(1-m_{1} m_{2} X\right)}{B_{m_{1}}\left(m_{2} X\right) B_{m_{2}}\left(m_{1} X\right)}\right)^{1 / m_{1} m_{2}}\right] .
\end{aligned}
$$

Using the integrality of $B_{\mathscr{M}}(X)$ and the same argument as at the end of $\S 2$, we can show that each of the four expressions in square brackets is a power series with integral coefficients (and similarly for $s \geq 3$ ). This generalizes the integrality statement of Theorem 1.

It remains to prove the integrality of $B_{\mathscr{M}}(X)$ for any set $\mathscr{M}$. But this follows easily from the corresponding statement for $|\mathscr{M}|=1$. Indeed, a wellknown and easily proved integrality criterion says that a power series of the form $\exp \left(\sum_{n=1}^{\infty} \beta(n) X^{n} / n\right)$ has integral coefficients if and only if the coefficients $\beta(n)$ are integers such that $\beta(n) \equiv \beta(n / p)\left(\bmod p^{\nu}\right)$ whenever $p^{\nu} \mid n$, 
$\nu \geq 1$. The integrality of $B_{m}(X)$ implies that the numbers $\beta(n)=\beta_{m}(n)$ satisfy these congruences. It follows that the numbers $\beta(n)=\beta_{\mathscr{M}}(n)$ also do and hence that $B_{\mathscr{M}}(X) \in \mathbb{Z}[[X]]$.

All of the contents of this section except for the statements about integrality can be found essentially in [2] (Corollary 4, Prop. 3, and their proofs).

\section{ACKNOWLEDGMENT}

The first author would like to thank James L'Heureux of West Chester University, one of whose questions led him to work on this paper.

\section{BIBLIOGRAPHY}

1. S. Gurak, Minimal polynomials for Gauss circulants and cyclotomic units, Pacific J. Math. 102 (1982), 347-353.

2. __ Minimal polynomials for circular numbers, Pacific J. Math. 112 (1984), 313-331.

3. D.H. Lehmer and E. Lehmer, Cyclotomy with short periods, Math. Comp. 41 (1983), 743-758.

Department of Mathematics, West Chester University of Pennsylvania, West Chester, Pennsylvania 19383

E-mail address: sgupta@wcu.bitnet

Max-Planck-Institut für Mathematik, Gottfried-Claren-Strasse 26, D-5300 Bonn 3, GERMANY

E-mail address: zagier@mpim-bonn.mpg.de 